\title{
Rhythmic oscillations of $\alpha$-amylase protein and its enzymatic activity levels in Drosophila melanogaster (Diptera: Drosophilidae)
}

\author{
Marcin A. CiUK, Piotr BEBAS and Bronislaw CYMBOROWSKI*
}

Department of Animal Physiology, University of Warsaw, Poland; e-mail: bron@biol.uw.edu.pl

Key words. Drosophilidae, Drosophila melanogaster, $\alpha$-amylase, rhythms, biological clock, insect

\begin{abstract}
In this report, we show that $\alpha$-amylase activity is rhythmic in the wild-type fruit fly Drosophila melanogaster, and that this rhythm exhibits the properties of a clock output. Moreover, the rhythm of amylase activity is accompanied by fluctuations in the Amy protein level under 12L : 12D conditions. A strong sexual dimorphism is evident in the oscillations of Amy protein and enzymatic activity. Under light : dark (LD) conditions on the control diet, CantonS wild-type Drosophila melanogaster exhibit a bimodal rhythm of amylase activity, particularly of the $\mathrm{AmyD}^{3}\left(\mathrm{Amy}^{3}\right)$ isoform, with morning and evening peaks. Under these conditions, Amy protein levels also oscillate significantly, again more strongly for the Amy ${ }^{3}$ isoform than $A m y^{1}\left(A_{m y}^{1}\right.$ ). A robust oscillation of $\mathrm{Amy}^{3}$ and Amy ${ }^{1}$ activity is also observed under DD conditions for both sexes. In constant light (LL) the rhythms dampen out, particularly in the males. A high level of dietary glucose causes an overall decrease in the amplitudes of the rhythmic oscillations of amylase activity, but the processes are nevertheless rhythmic, with peak activities at Zt 8 for the females, and at Zt0 for the males in LD. In constant darkness (DD) the rhythms are maintained. Mutants lacking a functioning oscillator, per ${ }^{01}$, exhibit a slight photoperiodicity in LD, with a decrease in amylase activity in both males and females during the late night in LD, but no rhythmic oscillations in DD.
\end{abstract}

\section{INTRODUCTION}

One of the reasons that physiological processes oscillate over time is to optimize expenditures of energy, a limited resource. An organism must be able to ingest and absorb enough nutrients of a quality sufficient to maintain its homeostasis. Given that feeding is a phenomenon dependent on the activity of the organism as a whole, it is logical that the production of digestive hydrolases should be temporally regulated to match feeding times. The question remains, whether these mechanisms are purely exogenous, or are they regulated by an endogenous clock mechanism.

The oscillations of clock components (clock gene transcripts and proteins) have been discovered in many tissues of $D$. melanogaster. This is the basis for the identification of so-called peripheral oscillators. Moreover, their autonomy has been unequivocally demonstrated, i.e. their ability to generate rhythms independently of the oscillator located in the brain. Their presence has been noted in the Malpighian tubules (Giebultowicz \& Hege, 1997), in the rectum, fat body and gastrointestinal tract (Giebultowicz, 2001). Since most of these tissues are closely connected to foraging, feeding, digestion and metabolism, it seems very logical that peripheral oscillators participate in the regulation of these physiological functions. Our knowledge of their $\operatorname{role}(\mathrm{s})$ in insects remains minimal. The connection between metabolic mechanisms and the biological clock is documented (Sarov-Blat et al., 2000; McDonald \& Rosbash, 2001; Ceriani et al., 2002). Most of these examples are enzymes

\footnotetext{
* Corresponding author
}

of intermediary metabolism, such as malate dehydrogenase, which participates in the citric acid cycle, or several enzymes involved in glycolysis, such as hexokinase, phosphoenolopyruvate dehydrogenase and transaldolase. This group also includes a number of transcripts with sequences similar to regions coding digestive enzymes, including L-maltase, $\mathrm{H}$-maltase, $\alpha$-glucosidase and $\alpha$-amylase-like. Thus, there potentially exist at least several rhythmically expressed genes which encode proteins regulating metabolism or participating in digestion. However, the rhythmic regulation of digestion has been poorly studied in insects, despite a number of reports that it occurs in other taxa, such as the rhythmic variation in ptyalin activity in rats (Vermouth et al., 1985; Bellavia et al., 1992; Ishikawa et al., 1993) and in humans (Bhoola et al., 1978; Jenzano et al., 1987; Rivera-Coll et al., 1993). Pancreatic amylase is also rhythmically secreted in rats (Maouyo et al., 1993) and in man (Dominguez-Munoz et al., 1998; Keller et al., 2001). More recently, it was demonstrated that not only are the canonical mammalian molecular oscillator proteins (PER1-3, CLOCK and REV-ERB) expressed with robust circadian rhythms in the human submandibular salivary glands, but that Amylase 1 is the sole salivary glucohydrolase which also cycles rhythmically in a robust fashion (Furukawa et al., 2005).

$\alpha$-amylase (synonyms: EC 3.2.1.1, 1,4- $\alpha$-D-glucanglucanohydrolase), is secreted by most if not all animals feeding on material containing polysaccharides composed of 1,4- $\alpha-\mathrm{D}$ linked glucose units (Svensson, 1988). In many insects, including Drosophila melanogaster, this 
enzyme is an important component of the digestive apparatus. Two genes encode $\alpha$-amylase in $D$. melanogaster, AmyP (Amylase-Proximal) and AmyD (Amylase-Distal), named so due to their distribution relative to the centromere of chromosome $2 \mathrm{R}$. The two genes are located some $5 \mathrm{~Kb}$ apart, and transcribed divergently, AmyP towards the centromere and $A m y D$ away from it. Interestingly, neither gene contains intron sequences (Gemmill et al., 1986; Doane et al., 1987). The two isoforms are further subdivided by the known electrophoretic variants they encode. For example, in the wild-type strain CantonS Amy genes encode variants $\mathrm{AmyP}^{1}$ and $\mathrm{AmyD}^{3}$, usually abbreviated as $A m y^{1}$ or $A_{m y}^{3}\left(A m y^{1,3}\right.$ for the whole genotype).

The regulation of $\alpha$-amylase in $D$. melanogaster is known largely in terms of differences between the expression of its activity in various strains of flies, and on various diets (Hickey \& Benkel, 1982; Hickey et al., 1994; Yamate \& Yamazaki, 1999). It has been shown that most strains usually express one or two amylase isoforms differing slightly in molecular mass and widely in their activity levels (Doane, 1967; Norman \& Doane, 1990). The differences in the activity levels of various isoforms of the enzyme are thought to be mostly caused by differences in protein levels rather than catalytic efficiency, but that the latter does make some contribution (Yamate \& Yamazaki, 1999). It has also been shown that $\alpha$-amylase is expressed in discrete portions of the midgut (Doane, 1969). Moreover, the expression of this enzyme varies in a strain-dependent manner and that in the posterior midgut it is age-dependent, at least in some strains.

Food quality is a powerful regulator of the expression of $\alpha$-amylase in Drosophila. The expression of this enzyme is strongly repressed by increased dietary glucose (Hickey \& Benkel, 1982; Magoulas et al., 1992, 1993; Thompson et al., 1992). The downregulation pertains to the rate of synthesis of the enzyme, since its mRNA level becomes altered, but the catalytic efficiency of individual enzyme molecules does not (Norman \& Doane, 1990). Catalytic efficiency does vary somewhat between strains of D. melanogaster (Yamate \& Yamazaki, 1999). A further report has shown that amylase in the head can be a marker for monitoring sleep deprivation and sleep drive both in flies and humans (Seugnet et al., 2006). These authors showed that total Amy activity and mRNA levels oscillate in total, whole-head Drosophila mRNA and in human salivary glands, and that they are strongly correlated to the rest state of the individual. No distinction was made in terms of which isoform was observed in D. melanogaster.

The present paper reports the rhythmic regulation of a process likely to be conditional to the digestion of polysaccharides in an insect maintained on a diet rich in starch. In this report we attempted to examine the nature of the rhythm of $\alpha$-amylase expression in the midgut of D. melanogaster. In particular, we wished to examine whether one or both of the Amy isoforms are expressed in rhythmic fashion, and at what level this regulation occurs. To additionally test the clock-control of amylase expression, we assayed total enzymatic activity in $\mathrm{per}^{01}$ insects lacking a functioning oscillator. We also decided to examine whether increased glucose levels can influence the phase of this rhythm.

\section{MATERIAL AND METHODS}

\section{Flies}

In this research we aimed to establish the characteristics of the rhythm of expression of $\alpha$-amylase in the midgut of the fruit fly Drosophila meloanogaster. We used the wild type strain, CantonS (CS) to determine the nature of the rhythm and as a control for further procedures. To examine whether the rhythm of $\alpha$-amylase expression is regulated by an endogenous clock(s), we used an arrhythmic mutant lacking a functional period gene, per $^{01}$. We selected CS as the wild type as the mutant stock is derived from this strain and expresses the same Amy ${ }^{1,3}$ isoforms. Additionally, $c A m y^{\text {null }}$ flies were used as negative controls where appropriate. All flies are maintained as cultures in our lab. The founding stock was obtained as a kind donation from Prof. J. Giebultowicz (OSU, Corvallis, OR), with the exception of the $c A m y^{\text {null }}$ stock, which was donated by Prof. W.W. Doane (Prof. Emeritus, School of Life Sciences, Arizona State University). The base culture and experimental control cultures were maintained in conditions of $12 \mathrm{~L}: 12 \mathrm{D}$, at $25^{\circ} \mathrm{C}$ and ca. $60 \%$ rel. humidity. All subsequent procedures and incubation of flies maintained under constant darkness (DD) and constant light (LL) conditions, were also performed at room temperature $25^{\circ} \mathrm{C}$. Time synchronised by an external time cue (LD) is referred to as Zeitgeber time, or Zt. Under constant conditions, subjective time is referred to as Circadian time, or $\mathrm{Ct}$.

\section{Experimental design}

The primary fruit fly culture is maintained on a standard cornmeal and agar diet. However, due to the unpredictable content of food-grade ingredients the experimental diets used reagentgrade ingredients from MP (formerly ICN, USA) and $\mathrm{POCH}$ (Poland). Because glucose is a strong repressor of $\alpha$-amylase activity in D. melanogaster, in addition to the control diet $(0.75 \%$ agarose, $5 \%$ each of starch, glucose and heat inactivated yeast) we examined the effect of a glucose diet (GD), which contained $5 \%$ by mass of glucose. Live yeast was not added to either of the diets.

Because both "central" and peripheral insect oscillators are thought to be subjected to photoperiodic regulation, it was necessary to examine the dietary variants under various photoperiods. The control flies were reared under an 12L : 12D photoperiod at $25^{\circ} \mathrm{C}$. To investigate the endogenous nature of the rhythm, flies were moved to free-running conditions (constant darkness, DD). Because continuous light (LL) is a well known desynchronising factor of insect biological oscillators, the procedures were also repeated under LL.

As sexual dimorphism in terms of energy requirements in $D$. melanogaster is quite pronounced, we decided to perform all procedures on males and females separately.

A day prior to eclosion, pupae were collected, sexed and grouped in vials containing appropriate media in batches of 10 . Eclosed insects were maintained under $12 \mathrm{~L}: 12 \mathrm{D}$ conditions for $24 \mathrm{~h}$. Immediately before the onset of day 2, the DD flies were transferred without exposure to light into DD conditions, whereas LL flies were transferred into LL conditions immediately following lights-on. The flies were kept under the new conditions for an additional $24 \mathrm{~h}$. Procedures which required working in the dark were performed under low intensity red light. 


\section{Collection of material}

Material was collected at 6 time-points throughout the day, Zeitgeber time $(\mathrm{Zt})$ or Circadian time (CT) 0, 4, 8, 12, 16 and 20 over at least three days. After a brief narcosis with $\mathrm{CO}_{2}$, the insects were transferred into $1.5 \mathrm{ml}$ tubes and frozen at $-80^{\circ} \mathrm{C}$. Immediately prior to zymography or Western blotting, the appropriate tubes were removed from the freezer and sharply rapped to decapitate, delimb and dewing the carcasses. The carcasses (abdomen and thorax) were transferred manually into a fresh tube. Total protein was extracted from each batch using a Sonoplus ultrasonic homogenizer (Bandelin, Germany), 1 carcass per $10 \mu \mathrm{l}$ of homogenization buffer: $10 \mathrm{mM}$ Tris- $\mathrm{HCl}, \mathrm{pH}$ 7.2 (Mendiola-Olaya et al., 2000), general purpose Protease Inhibitor Cocktail (PIC Sigma, USA) and 0.1\% Triton-X 100 (Merck, Germany). The homogenate was centrifuged at 18,000 $\mathrm{g}$ for $35 \mathrm{~min}$ at $4^{\circ} \mathrm{C}$. The supernatant was used in further procedures. Total protein was determined using the BCA protein assay (Pierce, USA). For spectrophotometric analyses, the crude protein extract was obtained from individual insects as above.

\section{Analysis of the results}

Where needed, electrophoretic band intensity was measured densitometrically using the ImageJ software package (National Institutes of Health, USA). The quantification of spectrophotometric data was performed on a 96-well microplate reader (Perkin-Elmer, USA) at $410 \mathrm{~nm}$. Quantified data were arranged for analysis and analyzed using Excel software (Microsoft). Data were translated into relative density or OD units, as appropriate, against the maximum value obtained for each data set. Overall statistical significance was assessed using a one-way ANOVA test at a significance level of $\mathrm{p}<0.05$. Post-hoc pairwise analyses were carried out using Tukey's HSD test, p < 0.05 .

\section{Zymography}

Starch zymography following PAGE in reducing conditions was used to examine the rhythm of $\alpha$-amylase activity. PAGE was performed according to a modification of a protease zymography protocol (Zeng et al., 2002), with a $10 \mu \mathrm{g}$ total protein sample from a homogenate of at least 8 insects loaded per well in $0.75 \mathrm{~mm}$ thick gels. In brief, a $10 \%$ separating polyacrylamide (Merck, Germany) gel containing $0.25 \%$ solubilised ACS potato starch (Amresco, USA) and no SDS was cast. A 4\% stacking gel, also without SDS was used. The running buffer and loading buffers were standard SDS-PAGE formulations (Laemmli, 1970). Following mixing with the loading buffer, the samples were heated to $50^{\circ} \mathrm{C}$ for $5 \mathrm{~min}$, briefly centrifuged, loaded onto the gel, and electrophoresed at $100 \mathrm{~V}$ for $15 \mathrm{~min}$ and at $170 \mathrm{~V}$ until completion. Following electrophoresis, the gels were incubated at $25^{\circ} \mathrm{C}$ in two $25 \mathrm{~min}$ changes of $\alpha$-amylase activity buffer, $50 \mathrm{mM}$ Tris- $\mathrm{HCl}, 30 \mathrm{mM} \mathrm{CaCl}_{2}, \mathrm{pH}$ 7.4 (Abraham \& Doane, 1978; Milanovic et al., 1989). The gels were then stained with Lugol's iodine, destained with lukewarm, running tap water and photographed with transmitted light on a tabletop scanner (Agfa, Germany). The enzymatic activity appears as light bands against a dark background.

\section{Western blotting}

Standard Laemmli SDS-PAGE was performed on $1.0 \mathrm{~mm}$ thick $10 \%$ acrylamide separating gels. The wells were loaded with a $50 \mu \mathrm{g}$ total protein sample from a homogenate of at least 8 insects. Human salivary amylase (Sigma, USA) was used as the positive control, $c A m y^{\text {mull }}$ total protein extracts were used as negative controls (data not shown). Following the electrophoresis, the transfers were performed overnight at $35 \mathrm{~V}$ and $4^{\circ} \mathrm{C}$ in a buffer containing $20 \%$ methanol and no SDS. After the transfer, the membranes were visually inspected using Ponceau Red staining. After destaining, the membranes were blocked for $2 \mathrm{~h}$ in 3\% skim milk suspended in PBS. Incubation was performed overnight in a $1: 500$ dilution of the stock antibody, rabbit antihuman salivary amylase (Sigma, USA), resuspended and aliquotted according to the manufacturer's instructions. On the following day, the blot was washed $4 \times 10 \mathrm{~min}$ in PBST (PBS containing $0.3 \%$ Tween-20), and incubated in the secondary antibody, alkaline phosphatase(AP)-conjugated anti-rabbit IgG in $3 \%$ milk for $1.5 \mathrm{~h}$ at RT. Following four washes in PBST and 1 in PBS, the blot was equilibrated in $0.1 \mathrm{M}$ Tris- $\mathrm{HCl} \mathrm{pH} 9.5$, then in $0.1 \mathrm{M}$ Tris- $\mathrm{HCl} \mathrm{pH} 9.5$ with $0.1 \mathrm{M} \mathrm{NaCl}$ and $0.05 \mathrm{M}$ $\mathrm{MgCl}_{2}$. The bands were visualised using NBT/BCIP as the substrate for AP in the latter buffer.

\section{Spectrophotometry}

Spectrophotometric analyses of total amylolytic activity were performed using $1 \mathrm{mM}$ pNp-maltopentoside (Sigma, USA) as a specific chromogenic substrate in amylase activity buffer (as described above) with the addition of PIC (as above). Briefly, each well was loaded with $5 \mu \mathrm{g}$ of total protein, and the volume was brought to $20 \mu 1$ with homogenization buffer (as above). Wells were loaded with $130 \mu \mathrm{l}$ of the substrate solution and incubated for $30 \mathrm{~min}$ at RT. At this point, the wells were scanned in a Victor-3 reader (Perkin-Elmer) in the same order and at the same intervals that they were loaded. Increased absorbance readings at $410 \mathrm{~nm}$ against a blank (substrate diluted to the same concentration with homogenization buffer but without protein) corresponded to the digestion of the artificial substrate by amylase and the release of free p-Nitrophenol.

\section{RESULTS}

Under control conditions, $12 \mathrm{~L}: 12 \mathrm{D}, 25^{\circ} \mathrm{C}$, in CantonS wild-type $D$. melanogaster females, $\mathrm{Amy}^{3}$ isoform activity is higher than $\mathrm{Amy}^{1}$ (Fig. 1A). Peak Amy ${ }^{3}$ activity occurs during the early night ( $\mathrm{Zt} \mathrm{16)}$, and reaches its minimum during the early day ( $\mathrm{Zt} 4)$. Using Tukey HSD post-hoc analysis (as in all cases below), it was shown that these changes are statistically significant, with a largest difference between the first half of the day and the first half of the night (Fig. 1C). No significant differences were observed in Amy ${ }^{1}$ activity in females. In males (Fig. 1B), the difference in activity level between $\mathrm{Amy}^{3}$ and Amy ${ }^{1}$ is even more pronounced. The rhythm of enzyme activity is also somewhat different in that it peaks slightly during early night, but troughs about $4 \mathrm{~h}$ later (Fig. 1D). The trough is significantly different from other daytime and nighttime values. In males, the activity of the Amy ${ }^{1}$ isoform shows a significant peak at $\mathrm{Zt} 8$ and a significant trough at $\mathrm{Zt} 20$. In females, Amy protein levels also vary with in a circadian manner (Fig. 1E), although the rhythm differs from the enzymatic activity. In CantonS females in $\mathrm{LD}$, the trough $\mathrm{Amy}^{3}$ protein level is phase delayed by $4 \mathrm{~h}$ in relation to the zymography, as is the peak (Fig. 1G). Although the amplitude of the variation of the Amy ${ }^{1}$ protein is large, and roughly follows the same pattern as that of $\mathrm{Amy}^{3}$, the changes are not statistically significant due to great individual variation. In males, the situation is somewhat different. Here, the peak and trough Amy protein levels are similar to the peaks and troughs of enzymatic activity, but with greater differences between them. $\mathrm{Amy}^{3}$ is expressed at a similar or lower level than Amy ${ }^{1}$ (Fig. 1F). In males, significant variation occurs in both 
A

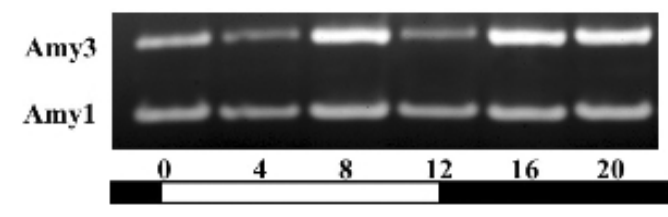

C

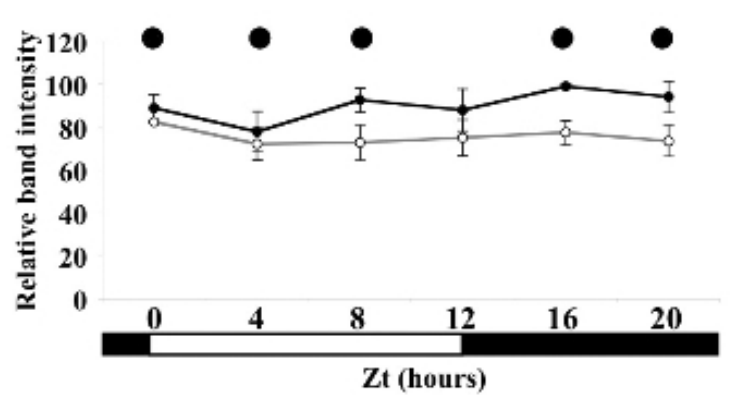

E

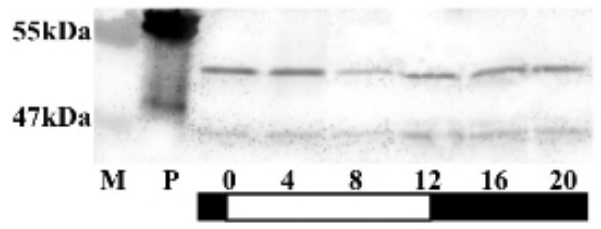

G

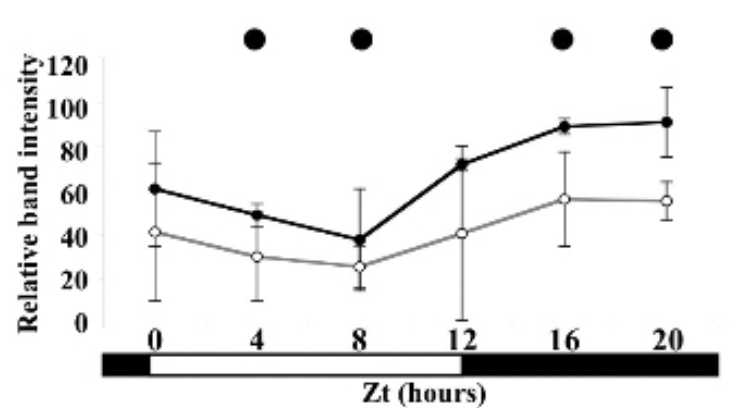

B

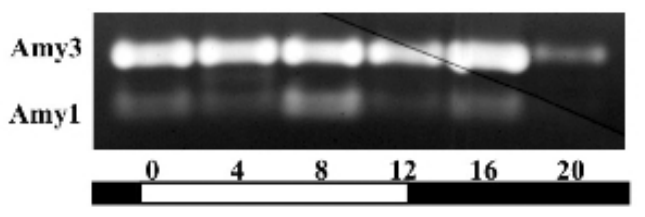

D

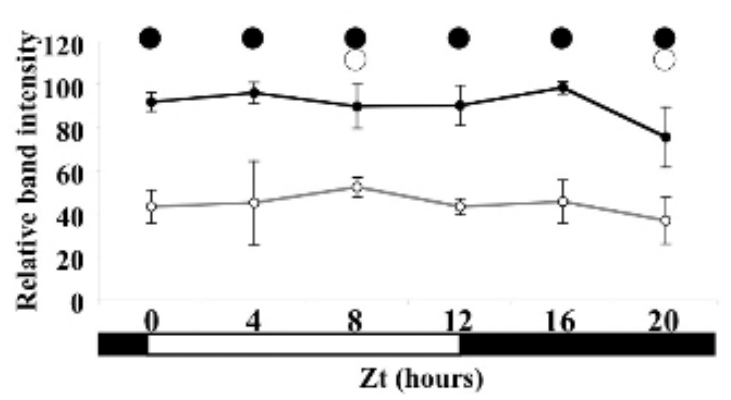

F

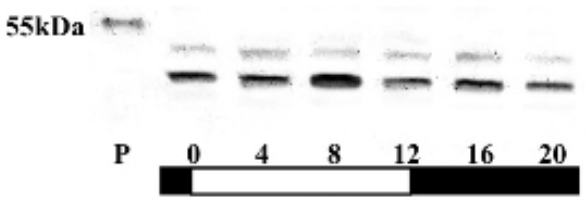

H

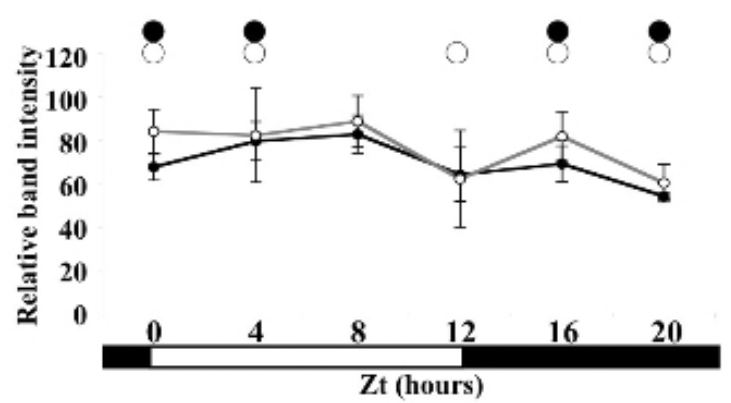

Fig. 1. Daily variation in the expression of Amy isoforms in wild-type D. melanogaster (CantonS), maintained on the control diet under L12 : D12 conditions: A - representative zymogram of females, B - representative zymogram of males, C - averaged densitometric analyses of 4 zymograms of females, D - averaged densitometric analyses of 4 zymograms of males, E - representative Western blot of changes in amylase protein levels of females, $\mathrm{F}$ - representative Western blot of changes in amylase protein levels of males, G - averaged densitometric analyses of 3 Western blots of females, D - averaged densitometric analyses of 4 Western blots of males. Grey lines with open circles indicate the Amy ${ }^{1}$ isoform. Black lines with black circles indicate the Amy ${ }^{3}$ isoform. Black and white bars below the $\mathrm{X}$ axis indicate lights off and lights on respectively. Black and open circles above the graphs indicate that the expression of the $\mathrm{Amy}^{3}$ and $\mathrm{Amy}^{1}$ isoform respectively at a particular time point differs significantly from at least one other time point (Tukey HSD). For further information see text. Error bars are \pm SD for that time point. Each lane of each gel contained a $10 \mu \mathrm{g}$ (Zymogram) or $50 \mu \mathrm{g}$ (Western Blot) sample of protein extract from 8-10 insects.

isoforms, with peaks during the late day and troughs during the late night (Fig. 1H).

Under DD conditions, both male and female wild-type D. melanogaster exhibit strong circadian variation, with evident sexual dimorphism. In females, the enzymatic activity level of $\mathrm{Amy}^{3}$ is slightly higher than that of $\mathrm{Amy}^{1}$ (Fig. 2A). However, Amy ${ }^{3}$ exhibits a very strong peak during the latter half of the subjective day and a slightly higher level during early day than at during the nighttime. No single, definite trough activity level is observed. Amy ${ }^{1}$ also exhibits a peak during the late day, differing significantly from the activity during the subjective dusk and late night (Fig. 2C). In the males, there is a higher level of amylase activity, with a different rhythm than in the females. The subjective day peak is protracted and spans a $4 \mathrm{~h}$ period from early to late subjective day (Fig. 2B and D). Although the amplitude of the changes in Amy activity is much lower than in the females, the two $\mathrm{Amy}^{3}$ activity peaks differ in a highly significant manner from all other time points. The troughs at the onset of subjective darkness and late subjective night differ from the peak points, where the latter also differs from subjective day break. The Amy ${ }^{1}$ activity levels exhibit an almost 
A

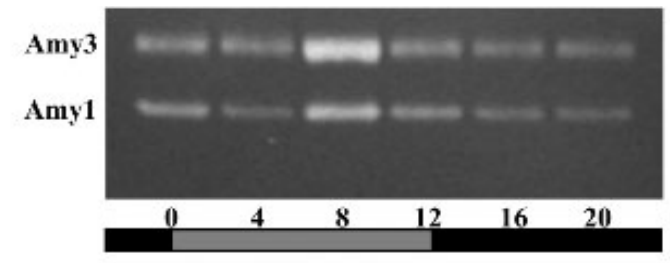

C

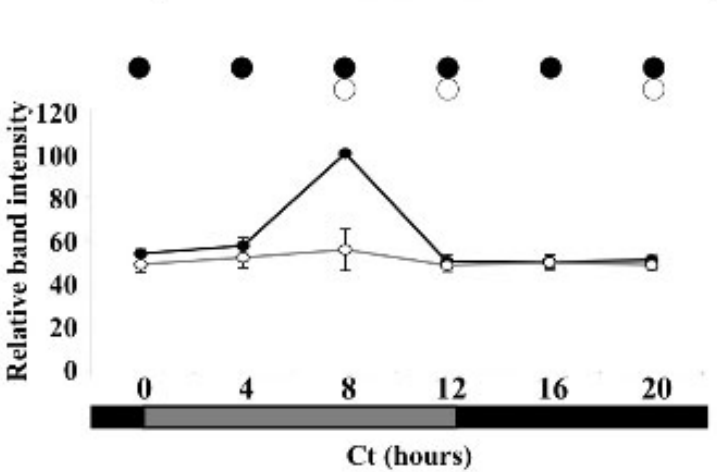

E

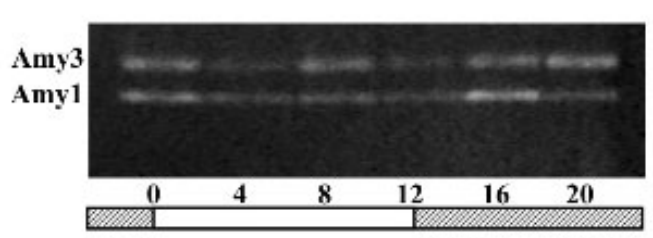

G

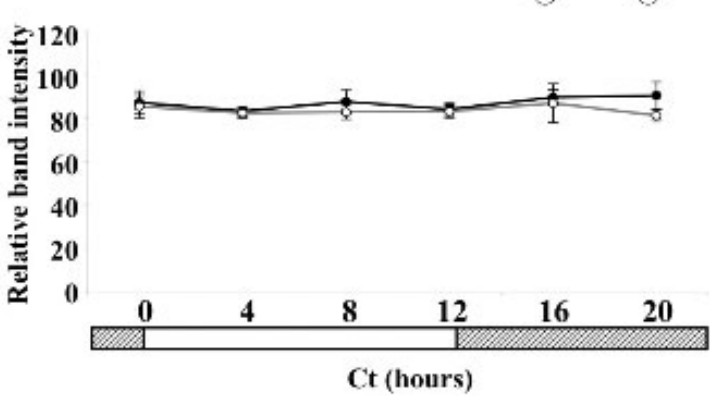

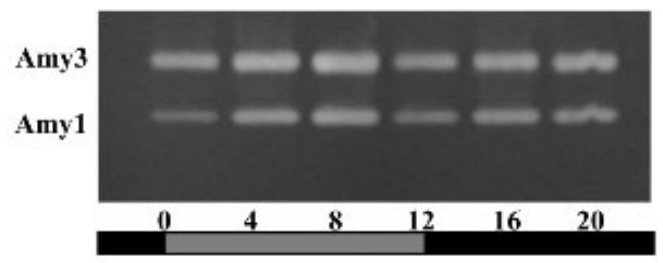

D

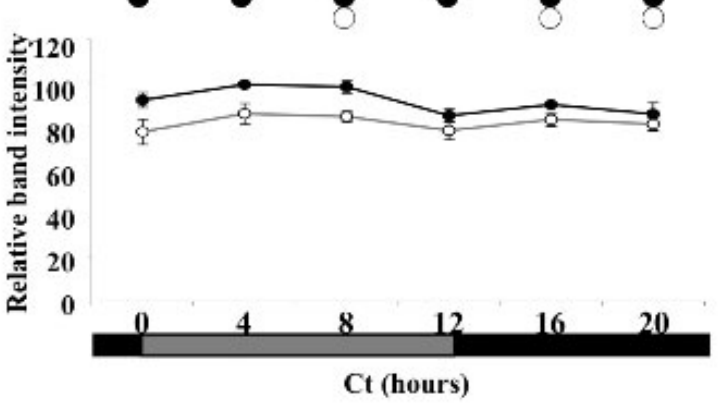

F

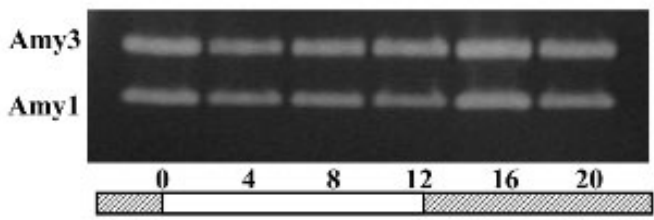

H

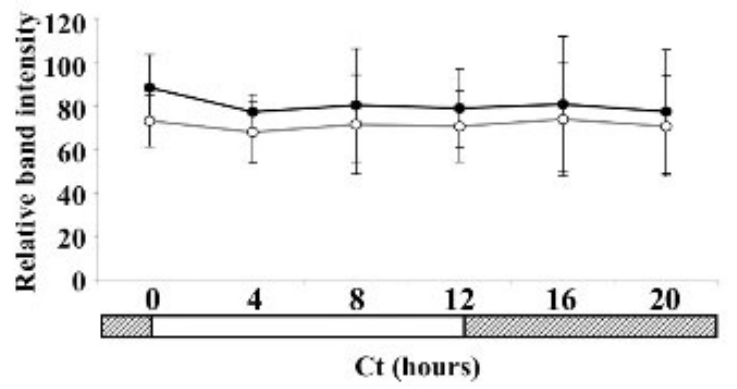

Fig. 2. Daily variation of the enzymatic activity of Amy isoforms in wild-type D. melanogaster (CantonS) maintained on the control diet: A, B - DD, representative zymograms of females and males respectively; C, D - DD, average values of densitometric analyses of 4 zymograms of females and males respectively; E, F - LL, representative zymograms of females and males respectively; G, H - LL, averaged densitometric analyses of 3 and 3 zymograms of females and males respectively. Grey lines with open circles indicate the Amy ${ }^{1}$ isoform. Black lines with black circles indicate the Amy ${ }^{3}$ isoform. Black and hatched bars below the $\mathrm{X}$ axis indicate subjective night, grey and white bars below the axis indicate subjective day in DD and LD respectively. Black and open circles above the graphs indicate that the expression of the $\mathrm{Amy}^{3}$ and $\mathrm{Amy}^{1}$ isoform respectively at a particular time point differs significantly from at least one other time point (Tukey HSD). For further information see text. Error bars are \pm SD for that time point. Each lane of each zymogram contained a $10 \mu \mathrm{g}$ sample of protein extract from $8-10$ insects.

identical pattern to $\mathrm{Amy}^{3}$, with an overall lower activity level with the same peak and trough times (Fig. 2D).

In LL conditions, in females, the rhythm becomes dampened out, although there are significant differences between the trough $\mathrm{Amy}^{3}$ activity levels during early subjective day and onset of the subjective night and the peak levels between time points 16 and 20. Amy ${ }^{1}$ activity shows no significant variation, except that there occurs a significant difference between early and late subjective night (Fig. 2E and G). Males exhibit no significant circadian variation in the activities of either isoform.
The high level of dietary glucose affects the pattern of Amy activity in the fruit fly. The activity of the usually dominant isoform, $\mathrm{Amy}^{3}$, is depressed relative to $\mathrm{Amy}^{1}$, particularly in the males. However, the activity of $\mathrm{Amy}^{3}$ continues to vary in a circadian manner (Fig. 3), both under LD and DD conditions. Under LD in the females $\mathrm{Amy}^{3}$ activity increases from $\mathrm{Zt} 4$ to $\mathrm{Zt8}$, where $\mathrm{Zt} 8$ is significantly different from the levels at $\mathrm{Zt0}$ and 4 (Fig. 3C). This rhythm is reminiscent of flies maintained under DD conditions on the control diet (Fig. 2C). The Amy ${ }^{1}$ isoform exhibits no significant variation in the females in LD. In the males on the glucose diet under LD conditions 
A

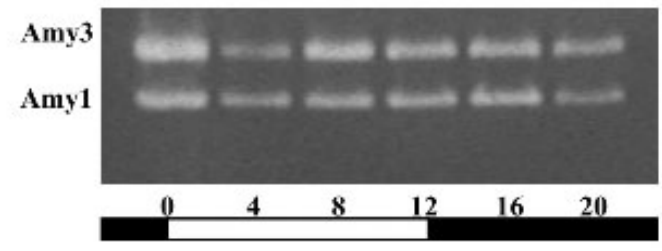

C

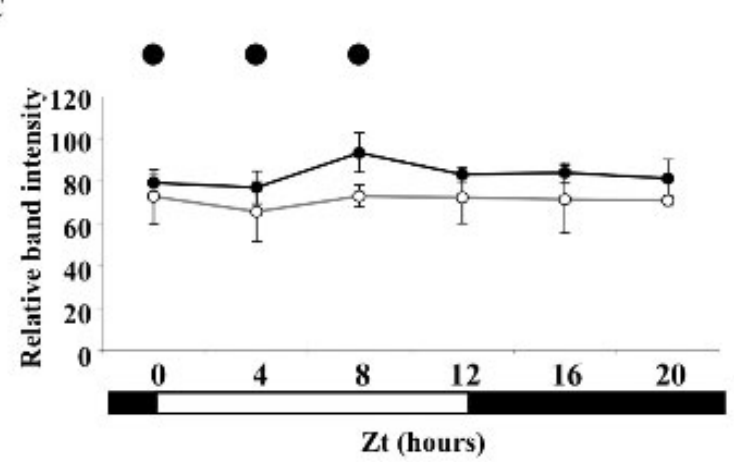

E

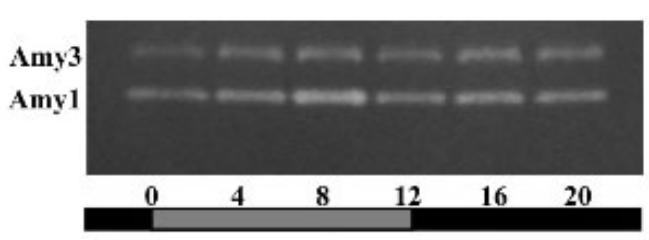

G

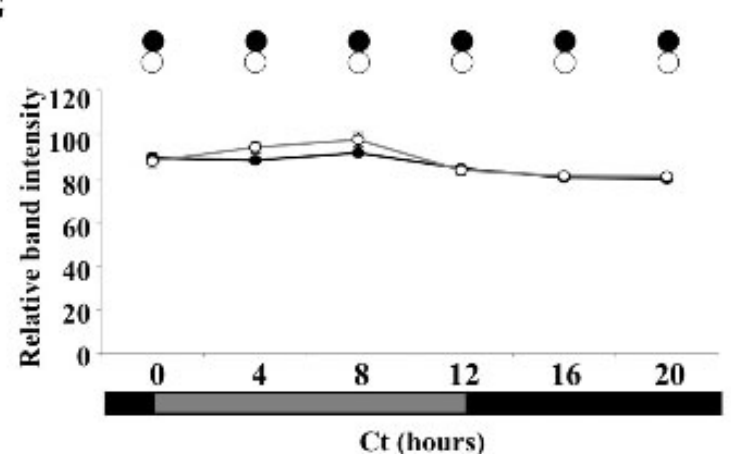

B

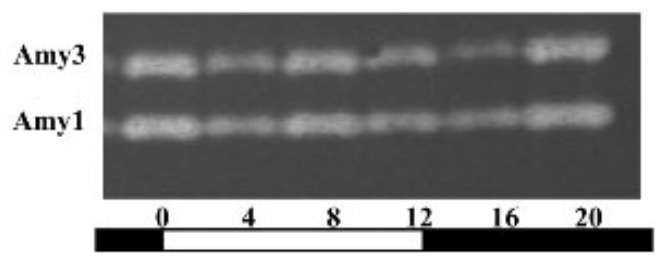

D

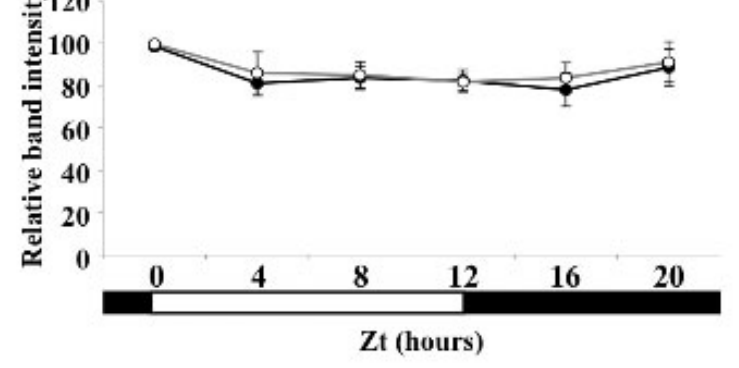

F

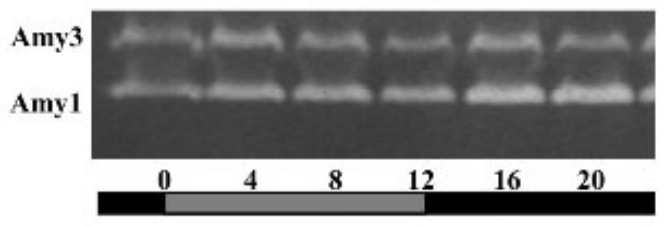

H

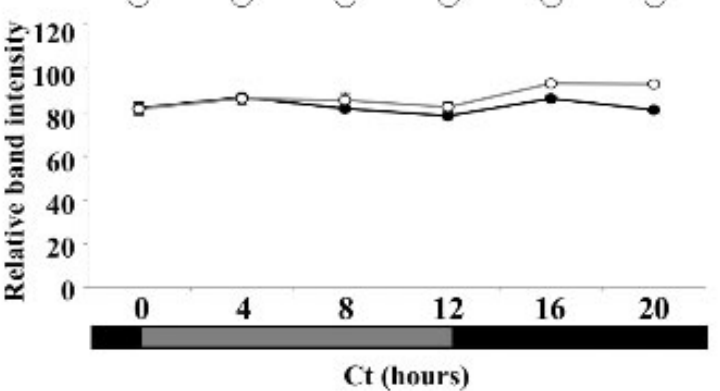

Fig. 3. Daily variation of the enzymatic activity of the Amy isoforms in wild-type D. melanogaster (CantonS) maintained on the glucose diet: A, B - LD, representative zymograms of females and males respectively; C, D - LD, average values of densitometric analyses of 4 and 3 zymograms of females and males respectively; E, F - DD, representative zymograms of females and males respectively; G, H - DD, average values of densitometric analyses of 3 and 3 zymograms of females and males respectively. Grey lines with open circles indicate the Amy ${ }^{1}$ isoform. Black lines with black circles indicate the Amy ${ }^{3}$ isoform. Black bars below the $\mathrm{X}$ axis indicate night or subjective night, white and grey bars below the axis indicate day or subjective day. Black and open circles above the graphs indicate that the expression of the $\mathrm{Amy}^{3}$ and $\mathrm{Amy}^{1}$ isoform respectively at a particular time point differs significantly from at least one other time point (Tukey HSD). For further information see text. Error bars are \pm SD for that time point. Each lane of each gel contained a $10 \mu \mathrm{g}$ sample of protein extract from $8-10$ insects.

(Fig. 3B and D), significant variation is observed between the peak $\mathrm{Amy}^{3}$ activity at daybreak and the following four time points (Fig. 3D). Interestingly, this also coincides with the peak Amy ${ }^{1}$ activity, which differs significantly from the trough Amy ${ }^{1}$ activity during the early night. This rhythm is maintained under DD conditions on the glucose diet, and the females (Fig. 3E and G) continue to exhibit a similar rhythm, although the differences in amplitude are much smaller. In the case of $\mathrm{Amy}^{3}$ activity, once again the peak value occurs during late subjective day, and is significantly different from the other subjective daytime points and from the subjective nighttime points. There is also a significant difference between the subjective day activity levels and the subjective night values (Fig. 3G). Amy ${ }^{1}$ activity is somewhat higher than that of $\mathrm{Amy}^{3}$, with a more defined peak during the late day. The pattern of peak and trough activities have a high degree of significance except for the difference between early and late subjective day. A similar significance between subjective day and night values is also observed. In the males (Fig. $3 \mathrm{~F}$ and $\mathrm{H}$ ), the rhythm occurs in a similar phase as in flies reared on the control diet, although Amyl activity is overall slightly higher than $\mathrm{Amy}^{3}$ activity (Fig. 3F). The $\mathrm{Amy}^{3}$ activity level at $\mathrm{Ct} 4$ differs significantly from all other time points except $\mathrm{Ct} 16$. The trough value at $\mathrm{Ct} 12$ is also significant. There is a difference in Amy ${ }^{1}$ activity 
A

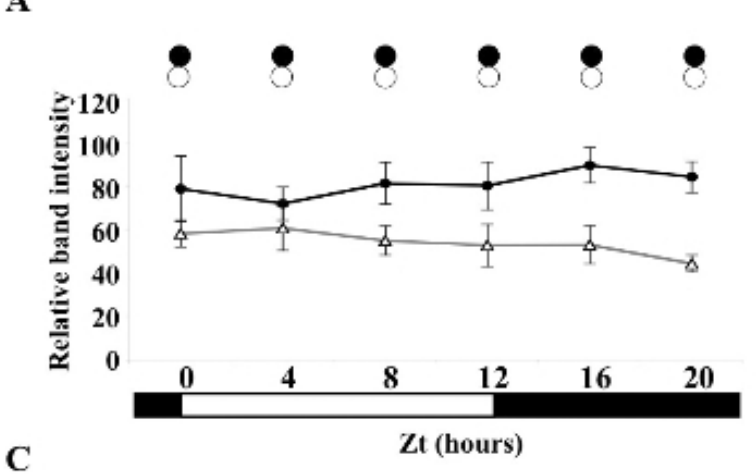

C

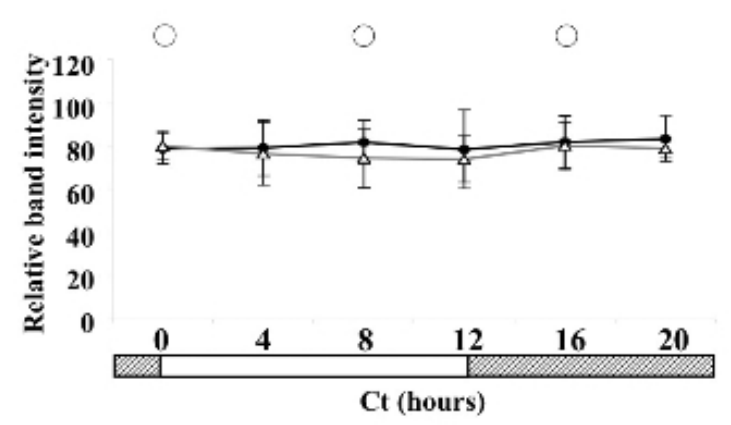

D

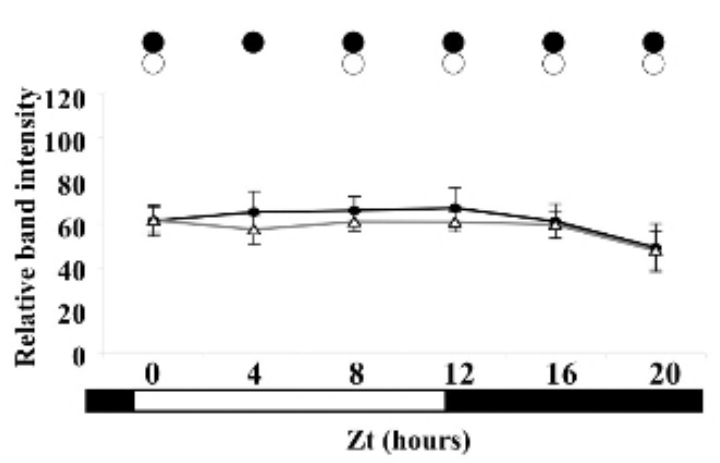

B

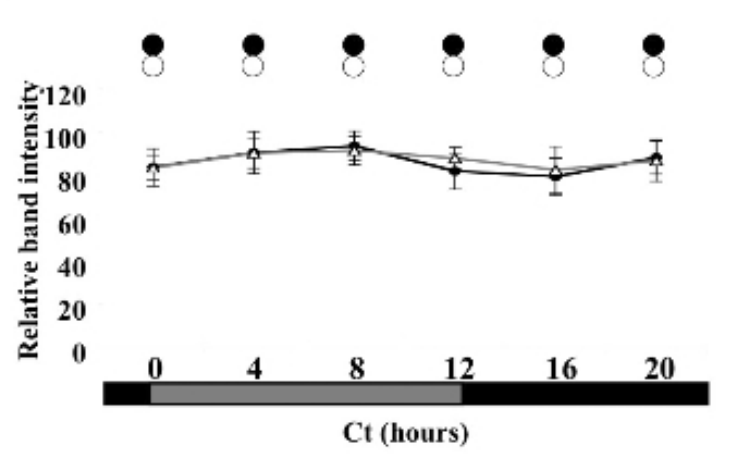

$\mathbf{E}$

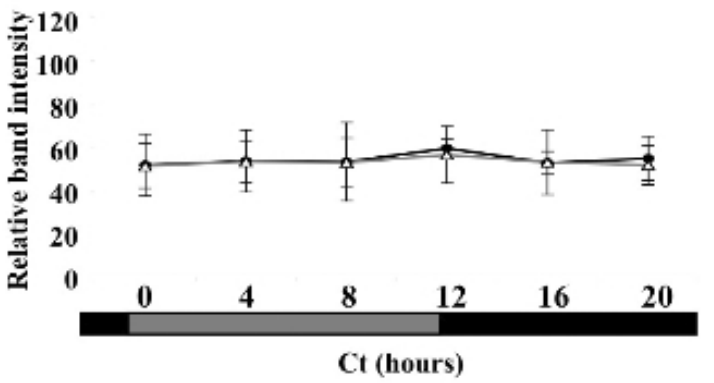

Fig. 4. Daily variation of the total amylolytic activity in D. melanogaster, maintained on the control diet, average activity of females (black lines with black circles) and males (grey lines with open triangles) of CantonS wild-type insects and per ${ }^{01}$ oscillator gene mutants: A - CantonS 12L : 12D, B - CantonS DD, C - CantonS LL, D - per ${ }^{01}$ LD, E - per ${ }^{01}$ DD. Bars below the X axis indicate as follows: (LD) black bars - lights-off, white bars - lights-on, (DD) black bars - subjective night, grey bars - subjective day, (LL) hatched bars - subjective night, white bars - subjective day. Black and open circles above the graphs indicate that the expression of amylase in females and males respectively at a particular time point differs significantly from at least one other time point (Tukey HSD). For further information see text. Error bars are \pm SD for that time point. Each measurement consisted of $5 \mu \mathrm{g}$ of total protein extracted from an individual insect. Each time point value is an average of least 12 separate measurements of individual insects.

levels between the peak at subjective dusk $(\mathrm{Ct12})$ and the peak at occurring some $4 \mathrm{~h}$ later (Fig. 3F). The subjective day values differ significantly from the subjective night values but not between each other.

The total Amy enzymatic activity pattern is similar to that measured by PAGE zymography (Figs 1A to D and Fig. 2 vs. Fig. 4), but better illustrates the nature of the differences between the sexes. It can be seen that although both sexes display a rhythm in LD, the female acrophase falls during the first third of the night whereas in the males it peaks at around $\mathrm{Zt} 4,12 \mathrm{~h}$ earlier (Fig. 4A). Thus the two rhythms are in exactly opposite phase and are highly significant. It is noteworthy that the total amy- lase activity per $\mu \mathrm{g}$ of total protein extract in females is on average substantially higher than in the males.

In DD, total amy activity in the females (Fig. 4B) reaches an acrophase at $\mathrm{Ct} 8$, with a strongly defined trough phase at $\mathrm{Ct} 16$ and $\mathrm{Ct} 4$. In the males, the activity peak is shifted in relation to the females and occurs at Ct12. The males do not exhibit a clearly defined trough phase, but instead there are two flattened troughs of amylase activity at $\mathrm{Ct} 0$ and $\mathrm{Ct} 16$ (Fig. 4B).

In LL, the females exhibit no discernible rhythm of total enzymatic activity (Fig. 4C). In the males, a slight trough is observed at $\mathrm{Ct} 8$ which is significantly different from the slight peaks at $\mathrm{Ct} 0$ and $\mathrm{Ct16}$ (Fig. 4C). 
Although $\mathrm{per}^{01}$ flies do not posses a functioning molecular oscillator, a slight photoperiodic response has been noted in them in the past (Helfrich-Forster, 2001). This can be observed in both males and females in LD (Fig. 4D), where we observed a decrease in amylase activity during the late night. This decrease differs significantly from the remaining time points. Under DD conditions, however, the oscillations dampen out almost completely and no significant changes in amplitude are observed (Fig. 4E).

\section{DISCUSSION}

In this report, we show that $\alpha$-amylase activity is rhythmic in wild-type $D$. melanogaster, and that this rhythm exhibits the properties of a clock output. Moreover, the rhythm of amylase enzyme activity is accompanied by fluctuations in the Amy protein level under 12L : $12 \mathrm{D}$ conditions. Lastly, a strong sexual dimorphism is evident in the oscillations of Amy protein and enzymatic activity. In LD, the activity of the Amy isoforms Amy ${ }^{1}$ and $\mathrm{Amy}^{3}$ varies in a circadian manner. However, only $\mathrm{Amy}^{3}$ shows robust oscillations in activity level in both males and females. The Amy ${ }^{1}$ isoform, although it seems to vary with a similar rhythm, demonstrates few significant changes, and only in the males (maximum at $\mathrm{Zt} 8$ vs. minimum at $\mathrm{Zt20}$ ). The aforementioned sexual dimorphism is evident in the rhythm of Amy activity in LD. Although both sexes exhibit a maximum level of $\mathrm{Amy}^{3}$ activity at $\mathrm{Zt16}$, the male trough phase occurs barely $4 \mathrm{~h}$ later, whereas the lowest level of activity in the females is observed around $\mathrm{Zt} 4$, thus during the day. Bimodality of the locomotor activity rhythm of $D$. melanogaster maintained in LD has been described previously (HamblenCoyle et al., 1992; Helfrich-Forster, 2000). As these authors showed, evening (E) and morning (M) peaks of locomotor activity in LD are present in both males and females. The enzymatic activity rhythm seems to be correlated with this pattern, though somewhat delayed since in both sexes the evening peak of amylase activity occurs some $4 \mathrm{~h}$ after lights-off (Zt16). The morning $\mathrm{Amy}^{3}$ activity peak in the males and females exhibits nearmaximum values of enzymatic activity at $\mathrm{Zt} 0$, slightly delayed with respect to the presumed $\mathrm{M}$ peak of locomotor activity (Helfrich-Forster, 2000). This seems to be a local maximum in the females, and the minimum occurs $4 \mathrm{~h}$ later. In the males, however, the activity is maintained at a high level throughout the day with only slight, but significant, variations. This is also likely concurrent with the feeding rhythm which is presumably synchronised with the locomotor activity rhythm (Oishi et al., 2004). Amy ${ }^{1}$ activity shows little significant variation in females. The total amylase activity pattern throughout the day in LD is quite similar to that observed using zymography. It nevertheless shows that in LD the activity in the males occurs at a lower level than in the females, and that the $\mathrm{E}$ peak of total amylase activity is not readily apparent in the males.

Interestingly, in terms of observed Amy protein levels, in the females both isoforms dip to a minimum during the latter part of the day in $\mathrm{LD}$, particularly $\mathrm{Amy}^{3}$. The observed rhythm is unimodal with a maximum during the latter half of the night. In the males, however, the oscillation of protein levels retains its bimodality, although the less active isoform (Amy $\left.{ }^{1}\right)$ seems to be expressed at a higher level of protein than the more active one $\left(\mathrm{Amy}^{3}\right)$. Because both techniques make use of PAGE electrophoresis in the presence of SDS and $\beta$-mercaptoethanol in the loading buffer and both are heated, both isoforms migrate in proportion to their size and thus to each other, and charge differences which may have reversed the bands in a completely native gel were negated. It is noteworthy that the oscillation pattern of protein levels is much more reminiscent of the enzymatic activity pattern in the males than in the females, suggesting that other, likely posttranslational regulatory mechanisms play a role in determining the activity rhythm (Yamate \& Yamazaki, 1999) in addition to the suspected regulators of Amy isoform transcription.

Under free-running conditions, the differences in Amy activity in wild type flies are even more robust. In the females, there is a single, high amplitude peak of $\mathrm{Amy}^{3}$ activity at $\mathrm{Ct} 8$, during the subjective day. Although at a much lesser amplitude, Amy ${ }^{1}$ activity in the females also demonstrates a peak at the same time. The males show an $\mathrm{Amy}^{3}$ activity peak during the subjective day (Ct4-8), which is more protracted but of lesser amplitude than that of the females. This rhythm is mirrored at a lower intensity of overall activity by the Amy ${ }^{1}$ isoform. HelfrichForster (2000) shows that males retain the evening (E) peak of locomotor activity in DD with a much smaller morning (M) peak. This was not observed in amylase activity in the males. The apparent reduction of the $M$ peak and the phase shift of the E peak towards the middle of the subjective day in the females is, however, concurrent with the locomotor results for CantonS females. Moreover, just as in the locomotor rhythm, the female $\mathrm{Amy}^{3}$ activity rhythm in DD is much more robust than in the males and also than in LD. Total amylase activity oscillations are rhythmic in DD, in phase with those assayed via zymography, but the amplitude of the oscillations is very small (see Results). This is indicative of some form of interaction between the two isoforms acting in a homogenous solution (Hickey \& Benkel, 1982), though it remains unknown whether it is directly between enzyme molecules, or whether another factor(s) is or are involved (Yamate \& Yamazaki, 1999).

We also assayed total activity in males and females in an arrhythmic mutant, per $^{01}$. Under LD conditions, both males and females showed a significant trough in Amy activity at $\mathrm{Zt} 0$. We observed no significant differences between the other time points. This one significantly lower level of activity may be a product of the weak circadian periodicity of per $^{01}$ flies, which has previously been demonstrated for locomotor activity rhythms in this mutant under LD (Helfrich-Forster, 2001). Under DD conditions, per $^{01}$ mutants did not exhibit any significant circadian variation in the total amylase activity, although high individual variation was observed. As mentioned, 
DD conditions allow the molecular clock to run freely and overtly exhibit endogenous rhythms otherwise continually synchronized by external time cues, especially the light/dark cycle. As such a rhythm is not observed under these conditions in clock-deficient insects, this is a likely indication that at least one peak of Amy activity needs a functioning circadian system to occur or, more generally, it is regulated by some output of the circadian clock. The strong sexual dimorphism of CantonS rhythms was not apparent in this mutant either. This is an indication, that a functioning clock is necessary for the sexspecific regulation of Amy activity. As mentioned, this is likely an adaptation to the different metabolic requirements of both sexes and merits further investigation.

Interestingly, in the w/t females, some significantly different activity levels occur in LL, and not in the males. The fact that it is not significant in the males, but significant in the females could be indicative of at least partial synchronization by a different time-regulated mechanism, such as that which regulates oviposition. Particularly so, since oviposition often retains its rhythmicity under LL conditions (Sheeba et al., 2001). This is reflected in total amylase activity, where some significantly different points do occur, but overall the oscillations are very small and there is high inter-individual variability, both in the males and females.

The sexual dimorphism of the amylase activity rhythms in LD and DD is likely due to the varied metabolic requirements of the males and females. In CantonS females, the E activity peak of $\mathrm{Amy}^{3}$ in LD and the retained $\mathrm{E}$ peak in DD seem to be correlated with the oviposition rhythm of wild-type females (McCabe \& Birley, 1998; Sheeba et al., 2001). The observed E peak of Amy activity consistently occurs some $4 \mathrm{~h}$ after the oviposition peak described. Even though the amylase activity rhythm is not likely to be causally synchronized with oviposition, vitellogenesis and the deposition of storage glycoproteins are very energy intensive processes and it would be advantageous for the females to co-regulate digestion with energy metabolism.

Although glucose is an inhibitor of amylase activity in D. melanogaster (Hickey \& Benkel, 1982; Hickey et al., 1994), likely through cAMP as a metabolic state messenger (Magoulas et al., 1992), its high dietary level did not cause arrhythmia of amylase activity. In females under high dietary glucose conditions, the circadian variation of the $\mathrm{Amy}^{3}$ activity peaked relatively strongly at $\mathrm{Zt} 8$, the time point at which there was a slight peak in LD and on the control diet, but subsequently the differences in activity levels dampened out. The oscillation of Amy ${ }^{1}$ activity in the females on the glucose diet under LD was very similar to the control diet under LD. Moreover, the overall $\mathrm{Amy}^{3}$ activity pattern was more strongly affected by a high glucose level than Amy ${ }^{1}$. This is consistent with previous results, where the proximal isoform of the gene is less repressed than the distal one (Hickey \& Benkel, 1982). It is of interest to note that the Amy ${ }^{3}$ activity lost its evening peak under LD, which was observed in females reared on the control diet. Since locomotor activity is regulated by a dual oscillator system (see above), it is also possible that a dual oscillator system regulates Amy activity as well, with the E peak being synchronized by nutrients. The Zeitgeber for such a model of regulation could be dietary glucose and to a lesser extent other oligosaccharides (Hickey \& Benkel, 1982), which could influence the level of Amy activity over time via food uptake in synchrony with locomotor and feeding rhythms, and/or the release of oligosaccharides, again primarily glucose, from degraded starch and glycogen. One candidate for such a system would be the regulation of the starvation response by takeout, a circadian-regulated gene expressed in the head and antennae which is thought to be responsible for the synchronization of circadian rhythms of locomotor activity and feeding behavior, whose transcriptional activity could indirectly influence Amy (Sarov-Blat et al., 2000). Moreover, in both the males and females, the rhythms of Amy activity levels were more similar to those observed under DD conditions, with the males being phase advanced by about $8 \mathrm{~h}$ relative to LD, again indicative of the regulation of at least one peak of activity by dietary glucose. Under DD conditions, the glucose diet affected the insects in a similar fashion, in that the variation of the activities of the two isozymes in both sexes were similar to those observed under the control diet in DD, except that the $\mathrm{Amy}^{3}$ activity was inhibited to a greater degree relative to Amy ${ }^{1}$ activity. The females showed a single peak in both isoforms at Ct16, with higher activity during the subjective day, and the males showed a slight but significant bimodality with peaks at $\mathrm{Ct} 4$ and $\mathrm{Ct} 8$ for both isoforms. Males maintained under DD conditions and on the glucose diet retained a slight, but significant bimodality, similar to that observed in DD on the control diet, without the prominent phase shift observed in LD. This is also likely indicative of the interaction between an environmental (photoperiodic or dietary) and an endogenous (free running) regulatory system.

Future experiments include the use of starved insects to ascertain to what extent the rhythm of $\alpha$-amylase expression is nutrient dependent. It would also be valuable to perform the experimental variants at all levels of gene expression, including the examination of mRNA levels.

ACKNOWLEDGEMENTS. This work was sponsored in part by MNISW (Ministry of Science and Postsecondary Education) Grant: 2P04C 06527 to B. C.

\section{REFERENCES}

Abraham I. \& Doane W.W. 1978: Genetic regulation of tissuespecific expression of amylase structural genes in Drosophila melanogaster. Proc. Natl Acad. Sci. USA 75: 4446-4450.

Bellavia S.L., Sanz E.G., Sereno R. \& Vermouth N.T. 1992: alpha-amylase circadian rhythm of young rat parotid gland: An endogenous rhythm with maternal coordination. Arch. Oral Biol. 37: 429-433.

Bhoola K.D., Matthews R.W. \& Roberts F. 1978: A survey of salivary kallikrein and amylase in a population of schoolgirls, throughout the menstrual cycle. Clin. Sci. Mol. Med. (Suppl.) 55: $561-566$. 
Ceriani M.F., Hogenesch J.B., Yanovsky M., Panda S. Straume M. \& Kay S.A. 2002: Genome-wide expression analysis in Drosophila reveals genes controlling circadian behavior. J. Neurosci. 22: 9305-9319.

DoAnE W.W. 1967: Quantitation of amylases in Drosophila separated by acrylamide gel electrophoresis. J. Exp. Zool. 164: 363-377.

DoAne W.W. 1969: Amylase variants in Drosophila melanogaster: linkage studies and characterization of enzyme extracts. $J$. Exp. Zool. 171: 31-41.

Doane W.W., Gemmill R.M., Schwartz P.E., Hawley S.A. \& NoRman R.A. 1987: Structural organization of the alphaamylase gene locus in Drosophila melanogaster and Drosophila miranda isozymes. Curr. Top. Biol. Med. Res. 14: 229-266.

Dominguez-Munoz J.E., Bregulla M., Nelson D.K., Glasbrenner B., Sauerbruch T. \& Malfertheiner P. 1998: Independent cycles of exocrine pancreatic secretion, hormones and gastroduodenal motility in healthy fasting humans: reassessment of a complex partnership. Neurogastroenterol Motil. 10: $27-34$.

Furukawa M., Kawamoto T., Noshiro M., Honda K.K., Sakai M., Fujimoto K., Honma S., Honma K., Hamada T. \& Kato Y. 2005: Clock gene expression in the submandibular glands. J. Dent. Res. 12: 1193-1197.

Gemmill R.M., Schwartz P.E. \& Doane W.W. 1986: Structural organization of the Amy locus in seven strains of Drosophila melanogaster. Nucl. Acids Res. 14: 5337-5352.

Giebultowicz J.M. 2001: Peripheral clocks and their role in circadian timing: insights from insects. Philos. Trans. R. Soc. Lond. (B) 356: 1791-1799.

Giebultowicz J.M. \& Hege D.M. 1997: Circadian clock in Malpighian tubules. Nature 386: 664.

Hamblen-Coyle M., Wheeler D.A., Rutila J.E., Rosbash M. \& HALL J.C. 1992: Behavior of period-altered circadian rhythm mutants of Drosophila in light : dark cycles (Diptera: Drosophilidae). J. Insect Behav. 5: 417-446.

Helfrich-Forster C. 2000: Differential control of morning and evening components in the activity rhythm of Drosophila melanogaster-sex-specific differences suggest a different quality of activity. J. Biol. Rhythms 15: 135-154.

Helfrich-Forster C. 2001: The locomotor activity rhythm of Drosophila melanogaster is controlled by a dual oscillator system. J. Insect Physiol. 47: 877-887.

HiCKEY D.A. \& BENKEL B. 1982: Regulation of amylase activity in Drosophila melanogaster: Effects of dietary carbohydrate. Biochem. Genet. 20: 1117-1129.

Hickey D.A., Benkel K.I., Fong Y. \& Benkel B.F. 1994: A Drosophila gene promoter is subject to glucose repression in yeast cells. Proc. Natl. Acad. Sci. USA 91: 11109-11112.

IsHikawa Y., CHEN C. \& IsHIDA H. 1993: The effect of starvation on the diurnal variation of amylase secretion from rat parotid glands. Res. Exp. Med. (Berl.) 193: 255-262.

Jenzano J.W., Brown C.K. \& Mauriello S.M. 1987: Temporal variations of glandular kallikrein, protein and amylase in mixed human saliva. Arch. Oral Biol. 32: 757-759.

Keller J., Groger G., Cherian L., Gunther B. \& Layer P. 2001: Circadian coupling between pancreatic secretion and intestinal motility in humans. Am. J. Physiol. Gastrointest. Liver. Physiol. 280: 273-278.

LAEMMLI U.K. 1970: Cleaveage of structural proteins during the assembly of bacteriophage T4. Nature 227: 680-685.

Magoulas C., Loverre-Chyurlia A. \& Hickey D.A. 1992: Amylase cis-acting sequences mediate the alleviation of glu- cose repression by cAMP in Drosophila. Biochem. Cell Biol. 70: 751-757.

Magoulas C., Bally-Cuif L., Loverre-Chyurlia A., Benkel B. \& HiCKEY D. 1993: A short 5'-flanking region mediates glucose repression of amylase gene expression in Drosophila melanogaster. Genetics 134: 507-515.

Majouyo D., Sarfati P., Guan D., Morisset J. \& Adelson J.W. 1993: Circadian rhythm of exocrine pancreatic secretion in rats: major and minor cycles. Am. J. Physiol. 264: 792-800.

McCABE C. \& Birley A. 1998: Oviposition in the period genotypes of Drosophila melanogaster. Chronobiol. Int. 15: 119-133.

McDonald M.J. \& Rosbash M. 2001: Microarray analysis and organization of circadian gene expression in Drosophila. Cell 107: 567-578.

Mendiola-Olaya E., Valencia-Jimenez A., Valdes-Rodriguez S., Delano-Frier J. \& Blanco-Labra A. 2000: Digestive amylase from the larger grain borer, Prostephanus truncatus Horn. Comp. Biochem. Physiol. (B) 126: 425-433.

Milanovic M., Andjelkovic M. \& Stamenkovic-Bojic G. 1989: Adaptive significance of amylase polymorphism in Drosophila IV. A comparative study of biochemical properties of the alpha-amylase in Drosophila melanogaster, D. hydei, D. subobscura and D. busckii. Comp. Biochem. Physiol. (B) 93: 629-634.

Norman R.A. \& Doane W.W. 1990: Dosage compensation and dietary glucose repression of larval amylase activity in Drosophila miranda. Biochem. Genet. 28: 601-613.

Oishi K., Shiota M., Sakamoto K., Kasamatsu M. \& Ishida N. 2004: Feeding is not a more potent Zeitgeber than the lightdark cycle in Drosophila. Neuroreport 15: 739-743.

Rivera-Coll A., Fuentes-Arderiu X. \& Diez-Noguera A. 1993: Circadian rhythms of serum concentrations of 12 enzymes of clinical interest. Chronobiol. Int. 10: 190-200.

Sarov-Blat L., So W.V., Liu L. \& Rosbash M. 2000: The Drosophila takeout gene is a novel molecular link between circadian rhythms and feeding behaviour. Cell 101: 647-656.

Seugnet L., Boero J., Gottschalk L., Duntley S.P. \& Shaw P.J. 2006: Identification of a biomarker for sleep drive in flies and humans. Proc. Natl. Acad. Sci. USA 103: 19913-19918.

Sheeba V., ChandrasheKaran M.K., Joshi A. \& Sharma V.K. 2001: Persistence of oviposition rhythm in individuals of Drosophila melanogaster reared in an aperiodic environment for several hundred generations. J. Exp. Zool. 290: 541-549.

Svensson B. 1988: Regional distant sequence homology between amylases, alpha-glucosidases and transglucanosylases. FEBS Lett. 230: 72-76.

Thompson D.B., Treat-Clemons L.G. \& Doane W.W. 1992: Tissue-specific and dietary control of alpha-amylase gene expression in the adult midgut of Drosophila melanogaster. $J$. Exp. Zool. 262: 122-134.

Vermouth N.T., Sanz E.G. \& Bellavia S.L. 1985: Circadian rhythm of secretory and non-secretory proteins in parotid gland of rat. Acta Odontol. Latinoam. 2: 25-30.

YAMATE N. \& YAMAZAKI T. 1999: Is the difference in alphaamylase activity in the strains of Drosophila melanogaster with different allozymes due to transcriptional or posttranscriptional control? Biochem. Genet. 37: 345-356.

ZeNG F., Zhu Y. \& CoHen A. 2002: Partial characterization of trypsin-like protease and molecular cloning of trypsin-like precursor cDNA in salivary glands of Lygus lineolaris. Comp. Biochem. Physiol. (B) 131: 453-463.

Received May 18, 2009; revised and accepted June 26, 2009 